Supplementary Material 


\title{
Comparison of paired ROC curves through a two-stage test
}

\author{
WENBAO YU, EUNSIK PARK * \\ Department of Statistics, Chonnam National University, Gwangju 500757, South Korea \\ YUAN-CHIN IVAN CHANG \\ Institute of Statistics Science, Academia Sinica, Taipei 11529, Taiwan
}

December 13, 2013

In this supplement, we present proofs of theorems in the main paper.

\section{$1 \quad$ Proof of Theorem 2.2}

Proof. Without loss of generality, assume both markers are standardized in a way that scores of non-diseased subjects follow $N(0,1)$. That is, $\mu_{X}=\mu_{\tilde{X}}=0$ and $\sigma_{X}=\sigma_{\tilde{X}}=1$. Then by Equations (2.4) and (2.5),

$$
\begin{gathered}
A U C_{1}=\Phi\left(\frac{\mu_{Y}}{\sqrt{1+\sigma_{Y}^{2}}}\right), \quad A U C_{2}=\Phi\left(\frac{\mu_{\tilde{Y}}}{\sqrt{1+\sigma_{\tilde{Y}}^{2}}}\right), \\
s A U C_{1}=\Phi\left(\frac{\mu_{Y}-c}{\sqrt{1+\sigma_{Y}^{2}}}\right), \quad \text { and }, \\
s A U C_{2}=\Phi\left(\frac{\mu_{\tilde{Y}}-c}{\sqrt{1+\sigma_{\tilde{Y}}^{2}}}\right) .
\end{gathered}
$$

If declaration (a) follows, since $\Phi$ is a cdf and therefore an increasing function, we have

$$
\frac{\mu_{Y}}{\sqrt{1+\sigma_{Y}^{2}}}=\frac{\mu_{\tilde{Y}}}{\sqrt{1+\sigma_{\tilde{Y}}^{2}}}
$$

\footnotetext{
${ }^{*}$ To whom correspondence should be addressed. Email: espark02@chonnam.ac.kr
} 
and

$$
\frac{1}{\sqrt{1+\sigma_{Y}^{2}}}=\frac{1}{\sqrt{1+\sigma_{\tilde{Y}}^{2}}} .
$$

Immediately, we have $\sigma_{Y}=\sigma_{\tilde{Y}}$ and $\mu_{Y}=\mu_{\tilde{Y}}$, thus, the two markers are distributed in the same way and thus declaration (b) follows.

On the other hand, if declaration (b) follows,

$$
R O C_{1}(t)=\Phi\left(\frac{\mu_{Y}+\Phi^{-1}(t)}{\sigma_{Y}}\right) \quad \text { and } \quad R O C_{2}(t)=\Phi\left(\frac{\mu_{\tilde{Y}}+\Phi^{-1}(t)}{\sigma_{\tilde{Y}}}\right)
$$

for any $t \in[0,1]$. This holds if and only if $\sigma_{Y}=\sigma_{\tilde{Y}}$ and $\mu_{Y}=\mu_{\tilde{Y}}$. Thus, the two markers have the same distributions and declaration (a) follows.

\section{Proof of Theorem 2.3}

Before proving the theorems 2.3 and 2.4, we give the definition of generalized U-statistics, which can be found in many literatures, for example, Lehmann (1963) and Sen (1974). Let $X_{1}, \cdots, X_{n_{1}} ; Y_{1}, \cdots, Y_{n_{2}} ; \cdots$ be independent samples of size $n_{i}$ from distributions $F, G$, and so on. A generalized-U statistic is defined as

$$
U=\left(\begin{array}{c}
n_{1} \\
m_{1}
\end{array}\right)^{(-1)}\left(\begin{array}{c}
n_{2} \\
m_{2}
\end{array}\right)^{(-1)} \cdots \sum \psi\left(X_{\alpha_{1}}, \cdots, X_{\alpha_{m_{1}}} ; Y_{\beta_{1}}, \cdots, Y_{\beta_{m_{2}}} ; \cdots\right),
$$

where the summation extends over all possible sets of subscripts satisfying $1 \leq X_{\alpha_{1}}<X_{\alpha_{2}}<\cdots<$ $X_{\alpha_{m_{1}}} \leq n_{1} ; 1 \leq Y_{\beta_{1}}<Y_{\beta_{2}}<\cdots<Y_{\beta_{m_{2}}} \leq n_{2} ; \cdots$, and $\psi\left(x_{1}, \cdots, x_{m_{1}} ; y_{1}, \cdots, y_{m_{2}} ; \cdots\right)$ is a function symmetric in the x's, in the y's, and so on. Here, symmetric in the x's means that the function $\psi$ retains the same value at any two permutations of $x_{\alpha_{1}}, \cdots, x_{\alpha_{m_{1}}}$.

Proof. We rewrite $\widehat{A U C} C_{1}-\widehat{A U C} C_{2}$ and $s \widehat{A U C} C_{1}-s \widehat{A U C} C_{2}$ as follows;

$$
\begin{aligned}
\widehat{A U C} C_{1}-\widehat{A U C}_{2} & =\frac{1}{n_{1} n_{2}} \sum_{i=1}^{n_{1}} \sum_{j=1}^{n_{2}} \psi\left(Y_{i}, X_{j}\right)-\frac{1}{n_{3} n_{4}} \sum_{k=1}^{n_{3}} \sum_{l=1}^{n_{4}} \psi\left(\tilde{Y}_{k}, \tilde{X}_{l}\right) \\
& =\frac{1}{n_{1} n_{2} n_{3} n_{4}} \sum_{i=1}^{n_{1}} \sum_{j=1}^{n_{2}} \sum_{k=1}^{n_{3}} \sum_{l=1}^{n_{4}} \varphi^{(1)}\left(Y_{i} ; X_{j} ; \tilde{Y}_{k} ; \tilde{X}_{l}\right)
\end{aligned}
$$

and

$$
\begin{aligned}
s \widehat{A U} C_{1}-s \widehat{A U} C_{2} & =\frac{\sum_{i=1}^{n_{1}} \sum_{j=1}^{n_{2}} \psi\left(Y_{i}, X_{j}+\hat{\delta}\right)}{n_{1} n_{2}}-\frac{\sum_{k=1}^{n_{3}} \sum_{l=1}^{n_{4}} \psi\left(\tilde{Y}_{k}, \tilde{X}_{l}+\hat{\tilde{\delta}}\right)}{n_{3} n_{4}} \\
& =\frac{1}{n_{1} n_{2} n_{3} n_{4}} \sum_{i=1}^{n_{1}} \sum_{j=1}^{n_{2}} \sum_{k=1}^{n_{3}} \sum_{l=1}^{n_{4}} \varphi^{(2)}\left(Y_{i} ; X_{j} ; \tilde{Y}_{k} ; \tilde{X}_{l}\right) .
\end{aligned}
$$


Then, $\widehat{A U C} C_{1}-\widehat{A U C} C_{2}$ and $s \widehat{A U} C_{1}-s \widehat{A U} C_{2}$ are two special generalized-U statistics with $m_{i}=1$, $i=1, \cdots, 4$. We also note that

$$
E\left(\widehat{A U C} C_{1}-\widehat{A U C} C_{2}\right)=A U C_{1}-A U C_{2}
$$

and

$$
E\left(s \widehat{A U} C_{1}-s \widehat{A U} C_{2}\right)=s A U C_{1}-s A U C_{2},
$$

where the expectation is taken with respect to the distribution function $F, G, \tilde{F}$, and $\tilde{G}$. From the result of Lehmann 1963) (Appendix (ii)) about generalized-U statistics, $N^{1 / 2}\left(\widehat{A U C}_{1}-\widehat{A U C} \widehat{C}_{2}-\right.$ $\left.\left(A U C_{1}-A U C_{2}\right)\right)$ and $N^{1 / 2}\left(s \widehat{A U} C_{1}-s \widehat{A U} C_{2}-\left(s A U C_{1}-s A U C_{2}\right)\right)$ are asymptotically bivariate normal with mean zero and covariance matrix $\Sigma=\left(\sigma_{r s}\right)$ where

$$
\sigma_{r s}=\left(N / n_{1}\right) \xi_{1000}^{r s}+\left(N / n_{2}\right) \xi_{0100}^{r s}+\left(N / n_{3}\right) \xi_{0010}^{r s}+\left(N / n_{4}\right) \xi_{0001}^{r s}
$$

$\xi_{1000}^{r s}, \xi_{0100}^{r s}, \xi_{0010}^{r s}$, and $\xi_{0001}^{r s}$ are covariances between $\varphi^{(r)}\left(Y_{i} ; X_{j} ; \tilde{Y}_{k} ; \tilde{X}_{l}\right)$ and $\varphi^{(s)}\left(Y_{i} ; X_{j^{\prime}} ; \tilde{Y}_{k^{\prime}} ; \tilde{X}_{l^{\prime}}\right)$, $\varphi^{(r)}\left(Y_{i} ; X_{j} ; \tilde{Y}_{k} ; \tilde{X}_{l}\right)$ and $\varphi^{(s)}\left(Y_{i^{\prime}} ; X_{j} ; \tilde{Y}_{k^{\prime}} ; \tilde{X}_{l^{\prime}}\right), \varphi^{(r)}\left(Y_{i} ; X_{j} ; \tilde{Y}_{k} ; \tilde{X}_{l}\right)$ and $\varphi^{(s)}\left(Y_{i^{\prime}} ; X_{j^{\prime}} ; \tilde{Y}_{k} ; \tilde{X}_{l^{\prime}}\right)$, and $\varphi^{(r)}\left(Y_{i} ; X_{j} ; \tilde{Y}_{k} ; \tilde{X}_{l}\right)$ and $\varphi^{(s)}\left(Y_{i^{\prime}} ; X_{j^{\prime}} ; \tilde{Y}_{k^{\prime}} ; \tilde{X}_{l}\right)$, respectively for $i \neq i^{\prime}, j \neq j^{\prime}, k \neq k^{\prime}$, and $l \neq l^{\prime}$ for $r, s=1,2$.

\section{Proof of Theorem 2.4}

Proof. Rewrite $\widehat{A U C} C_{1}-\widehat{A U C} C_{2}$ and $s \widehat{A U} C_{1}-s \widehat{A U} C_{2}$ as follows;

$$
\begin{aligned}
\widehat{A U C} \widehat{A C}_{1}-\widehat{A U C} & =\frac{1}{n m} \sum_{i=1}^{n} \sum_{j=1}^{m} \psi\left(Y_{i}, X_{j}\right)-\frac{1}{n m} \sum_{i=1}^{n} \sum_{j=1}^{m} \psi\left(\tilde{Y}_{i}, \tilde{X}_{j}\right) \\
& =\frac{1}{n m} \sum_{i=1}^{n} \sum_{j=1}^{m} \varphi^{(1)}\left(W_{i} ; V_{j}\right)
\end{aligned}
$$

and

$$
\begin{aligned}
s \widehat{A U C} C_{1}-s \widehat{A U} C_{2} & =\frac{1}{n m}\left[\sum_{i=1}^{n} \sum_{j=1}^{m} \psi\left(Y_{i}, X_{j}+\hat{\delta}\right)-\sum_{i=1}^{n} \sum_{j=1}^{m} \psi\left(\tilde{Y}_{i}, \tilde{X}_{j}+\hat{\tilde{\delta}}\right)\right] \\
& =\frac{1}{n m} \sum_{i=1}^{n} \sum_{j=1}^{m} \varphi^{(2)}\left(W_{i} ; V_{j}\right) .
\end{aligned}
$$

Since $\left\{W_{i}=\left(Y_{i}, \tilde{Y}_{i}\right)\right\}$ and $\left\{V_{j}=\left(X_{j}, \tilde{X}_{j}\right)\right\}$ are independently generated from distributions $F$ and $G$, $\widehat{A U C} C_{1}-\widehat{A U C} C_{2}$ and $s \widehat{A U} C_{1}-s \widehat{A U} C_{2}$ are two special generalized-U statistics with $m_{i}=1, i=1,2$, $n_{1}=n$ and $n_{2}=m$. We also note that

$$
E\left(\widehat{A U C} C_{1}-\widehat{A U C} C_{2}\right)=A U C_{1}-A U C_{2}
$$


and

$$
E\left(s \widehat{A U C} C_{1}-s \widehat{A U} C_{2}\right)=s A U C_{1}-s A U C_{2},
$$

where the expectation is taken with respect to distribution functions $F$ and $G$. From the result of Lehmann 1963) (Appendix (ii)) about generalized-U statistics, $N^{1 / 2}\left(\widehat{A U C} C_{1}-\widehat{A U C} C_{2}-\left(A U C_{1}-\right.\right.$ $\left.\left.A U C_{2}\right)\right)$ and $N^{1 / 2}\left(s \widehat{A U} C_{1}-s \widehat{A U} C_{2}-\left(s A U C_{1}-s A U C_{2}\right)\right)$ are asymptotically bivariate normal with mean zero and covariance matrix $\Sigma=\left(\sigma_{r s}\right)$ where $\sigma_{r s}=(N / n) \xi_{10}^{r s}+(N / m) \xi_{01}^{r s}$. Here $\xi_{10}^{r s}, \xi_{01}^{r s}$ are covariances between $\varphi^{(r)}\left(W_{i} ; V_{j}\right)$ and $\varphi^{(s)}\left(W_{i} ; V_{j^{\prime}}\right), \varphi^{(r)}\left(W_{i} ; V_{j}\right)$ and $\varphi^{(s)}\left(W_{i^{\prime}} ; V_{j}\right)$, respectively for $i \neq i^{\prime}$ and $j \neq j^{\prime}$ for $r, s=1,2$.

\section{References}

Lehmann, E. L. (1963) Robust Estimation in Analysis of Variance. The Annals of Mathematical Statistics, 34(3), 957-966.

Sen, P. K. (1974) Weak convergence of generalized U-statistics. The Annals of Probability, 90-102. 